\title{
Adult's onset Still disease occurring during pregnancy: case-report and literature review
}

Léo Plaçais ${ }^{1}$, Arsène Mekinian ${ }^{1}$, Marie Bornes ${ }^{2}$, Armelle Poujol-Robert ${ }^{3}$, Naiké Bigé ${ }^{4}$, Olivier Fain $^{1}$.

${ }^{1}$ AP-HP, Hôpital Saint-Antoine, service de médecine interne and Inflammation-ImmunopathologyBiotherapy Department (DHU i2B), Sorbonne Universités, UPMC Univ Paris 06, F-75012, Paris, France

${ }^{1}$ AP-HP, Hôpital Tenon, service de gynécologie et d'obstétrique, UPMC Univ Paris 06, F-75012, Paris, France

${ }^{1}$ AP-HP, Hôpital Saint-Antoine, service de hépatologie, UPMC Univ Paris 06, F-75012, Paris, France

${ }^{4}$ AP-HP, Hôpital Saint-Antoine, service de réanimation médicale, UPMC Univ Paris 06, F-75012, Paris, France

\section{Letter to the editor}

Running title: Adult Still disease during pregnancy

Correspondence and reprint requests to: Arsene Mekinian, Hôpital Saint-Antoine, service de médecine interne and Inflammation-Immunopathology-Biotherapy Department (DHU i2B), Sorbonne Universités, UPMC Univ Paris 06, F-75012, Paris, France

Mail: arsene.mekinian@aphp.fr

Tel: 0(33)148026047

Fax: 0(33)148026393

Key words: AOSD, pregnancy, pregnancy-revealed AOSD

Conflicts of Interest and Funding Sources: none.

\section{Rheumatology key message}

Pregnancy-revealed AOSD is a specific subset with early pregnancy flares and impaired obstetrical outcome. 
SIR,

Adult onset Still's disease is a rare affection classified among non-hereditary autoinflammatory diseases. The clinical presentation goes from fever with arthralgia and maculopapular eruption to life-threatening manifestations such as secondary lymphohistiocytosis. The mechanisms of AOSD remains unclear, but seems implicate NK cells dysfunction with pro-inflammatory cytokines secretion including IL-1ß, IL-6 and IL-18 [1]. Several reports mentioned AOSD occurring during pregnancy, mostly in first and second trimester and there is still a debate on whether it could compromise or not the pregnancy outcome. We here report a case of AOSD revealed during pregnancy with a life-threatening presentation along with a review of 19 cases from literature.

A 38-years old woman was treated in our department for diffuse systemic sclerosis and associated Sjögren syndrome. Both diseases were quiescent at the time of the report and none required immunosuppressive therapy. In May 2016 she was pregnant at 12 weeks of gestation and presented with acute fever without any associated features. Laboratory data revealed mild liver cytolysis (AST and ALT $\mathrm{x} 4 \mathrm{~N}$ ) but a large screening for infectious and auto-immune diseases was negative and hepato-biliar imaging was normal. Fever and cytolysis spontaneously regressed in 3 days. Two month later, she was hospitalized again for intense fever, sore throat and abdominal pain. Laboratory analysis revealed liver cytolysis (ALT and AST x10N) with cholestasis, elevated bilirubin and increased C-reactive protein levels $(53 \mathrm{mg} / \mathrm{L})$. Ferritin plasma levels were slightly high $(657 \mathrm{ng} / \mathrm{mL})$. Extended screening for infection remained negative and symptoms spontaneously regressed and she was discharged without any treatment. One month later she was admitted again for fever and arthromyalgia at 28 weeks of gestation. Laboratory data showed increased C-reactive protein levels $(163 \mathrm{mg} / \mathrm{L})$ associated with liver cytolysis $(\mathrm{x} 2 \mathrm{~N})$, and ferritin levels at $371 \mathrm{mg} / \mathrm{l}$. Cervico-thoraco-abdominal imaging revealed mild non-compressive pericarditis and persistent homogenous hepatomegaly (22 $\mathrm{cm}$ of great axis). Because of persistent fever and polyarthralgias, after exclusion of active infection, steroids were started (prednisone $1 \mathrm{mg} / \mathrm{kg}$ ) associated with colchicine, which allowed clinical remission and C-reactive protein significant decrease. When decreasing steroids at $12.5 \mathrm{mg} /$ day, she again experienced fever and arthralgias recurrence and intravenous immunoglobulins were started. Just before intravenous immunoglobulins, acute agranulocytosis occurred and was considered related to colchicine. The agranulocytosis was complicated with severe sepsis from urinary tractus infection, so foetal extraction was decided and realized at 34 weeks of gestation. Despite foetal extraction and sepsis control, the fever and 
polyarthralgias persisted, associated with severe cholestasis (x15N) and C-reactive protein levels at $320 \mathrm{mg} / \mathrm{l}$. Ferritin levels were thus at $41000 \mathrm{ng} / \mathrm{mL}$ with glycosylated ferritin less than 5\%. The diagnosis of AOSD was stated according to Yamaguchi and Fautrel criteria [2;3] and steroids were associated with anakinra (100 mg/day) allowing rapid clinical and biological remission.

AOSD can be revealed by pregnancy as first suggested in a case report from 1982 [4] eleven years after the first disease's description. We gathered data about 19 additional cases from the literature of AOSD revealed during the pregnancy [Table 1]. All cases concerned women from 19 to 38 years old (median age 28.6). Upon 18 pregnancies, median term at first flare was 16.77 weeks of gestation. Most women were in their first and second trimester (from 8 to 26 weeks of gestation). Clinical features mostly included arthritis and arthromyalgia, hectic fever and pharyngitis. Ferritin levels ranged from 1311 to $41424 \mathrm{ng} / \mathrm{mL}$. Most patients required steroids (n=16/20), and two were treated by intravenous immunoglobulins. All patients had systemic involvement and none presented as a chronic articular form. There were no obvious differences between the monocyclic and polycyclic forms. Obstetrical complications were frequent $(n=11 / 20)$ mostly showing up as prematurity $(n=10 / 20)$ though ours was due to foetal extraction and three of them could be linked to pre-term premature rupture of membranes potentially induced by the steroid regimens. There were three cases with intrauterine growth restriction, two with oligohydroamnios and one with neonatal death. In pregnancy-revealed AOSD, flares seemed to appear in first and second trimester earlier than in known AOSD [5]. Obstetrical complications seemed to be frequent as 50\% of the pregnancies ended up with prematurity and $15 \%$ complicated by intra-uterine growth restriction even though some could be related to steroid therapy adverse events. The pathophysiological link isn't clear but recent studies showed high levels of IL18 during pregnancy [6] that could act as a trigger to a previously latent disease and be responsible for pregnancy-induced AOSD. The treatment of AOSD during pregnancy could be challenging. Steroids can be used, but may be associated with adverse events, like gestational diabetes, arterial hypertension. Intravenous immunoglobulins can be used if necessary in presence of life-threatening AOSD [7]. The use of other agents, like anakinra and tocilizumab cannot be recommended during pregnancy [8]. In conclusion, pregnancy-revealed AOSD appears to be a specifical subset of the disease with a systemic course, flares on first and second trimester, obstetrical complications such as prematurity and IUGR sometimes leading to life-threatening situations requiring parenteral corticotherapy and intravenous immunoglobulins. 


\section{REFERENCES}

ACCEPTED MANUSCRIPT

1. Anneleen Avaua et al, Cytokine balance and cytokine-driven natural killer cell dysfunction in systemic juvenile idiopathic arthritis. Cytokine \& Growth Factor Reviews, Volume 26, Issue 1, February 2015, Pages 35-45. DOI: 10.1016/j.cytogfr.2014.05.005

2. Yamaguchi M. et al, Preliminary criteria for classification of adult Still's disease. J Rheumatol. 19:424-30, 1992. PMID: 1578458

3. Fautrel B. et al, Proposal for a new set of classification criteria for adult-onset still disease. Medicine (Baltimore) 81:194-200, 2002. PMID: 11997716

4. Green J et al, Adult Still's disease associated with pregnancy. Isr J Med Sci 1982;18:1037-9. PMID: 7174271

5. Gerfaud-Valentin et al, Adult-onset Still's disease and pregnancy: about ten cases and review of the literature. Rheumatology International, June 2014, Volume 34, Issue 6, pp 867-871. DOI: 10.1007/s00296-013-2765-5.

6. Qin Wang et al, Metabolic profiling of pregnancy: cross-sectional and longitudinal evidence. BMC Med. 2016; 14: 205. DOI: 10.1186/s12916-016-0733-0

7. Liozon $\mathrm{E}$ et al, Intravenous immunoglobulins for adult Still's disease and pregnancy. Rheumatology. 1999;38(10):1024-1025. PMID: 10534562.

8. Levy $\mathrm{R}$ et al, Critical review of the current recommendations for the treatment of systemic inflammatory rheumatic diseases during pregnancy and lactation. Autoimmun Rev. 2016 Oct;15(10):955. DOI: 10.1016/j.autrev.2016.07.014 
Table 1. AOSD revealed during pregnancy: cases from the literature review.

\begin{tabular}{|c|c|c|c|c|c|c|c|}
\hline $\begin{array}{c}\text { Year / } \\
\text { Author }\end{array}$ & Age & $\begin{array}{l}\text { Time of } \\
\text { pregnancy } \\
\text { (wg) }\end{array}$ & $\begin{array}{c}\text { AOSD } \\
\text { features }\end{array}$ & $\begin{array}{c}\text { Ferritin } \\
\text { levels / } \\
\text { GF levels }\end{array}$ & Treatment & $\begin{array}{c}\text { AOSD } \\
\text { outcome } \\
\text { during the } \\
\text { pregnancies }\end{array}$ & $\begin{array}{c}\text { Adverse } \\
\text { Obstetrical } \\
\text { outcome }\end{array}$ \\
\hline $\begin{array}{l}\text { 1980, } \\
\text { Kaplinsky [1] }\end{array}$ & 34 & Unknown & $\underset{\mathrm{E}}{\mathrm{A}, \mathrm{F}, \mathrm{LN},}$ & NA/NA & $\begin{array}{l}\text { Aminopyrin } \\
\text { e, gold salt }\end{array}$ & $\begin{array}{l}\text { Polycyclic } \\
\text { (PP) }\end{array}$ & None \\
\hline $\begin{array}{l}\text { 1982, Green } \\
\text { [2] }\end{array}$ & 23 & $25 \mathrm{wg}$ & $\begin{array}{c}\text { A,F, } \\
\text { HSMG, } \\
\text { LN, E }\end{array}$ & NA/NA & NA & $\begin{array}{l}\text { Polycyclic } \\
(28 w g, \text { PP) }\end{array}$ & $\begin{array}{l}\text { Neonates } \\
\text { death, } \\
\text { prematurity } \\
\text { at } 28 \mathrm{wg}\end{array}$ \\
\hline $\begin{array}{l}\text { 1985, Yebra } \\
\text { Bango [3] }\end{array}$ & 19 & $12 \mathrm{wg}$ & $\begin{array}{c}\text { A,F, } \\
\text { HSMG, } \\
\text { E, Pha }\end{array}$ & NA/NA & Prednisone & Monocyclic & 0 \\
\hline 1990, Katz [4] & 32 & PP 2 m & $\mathrm{E}, \mathrm{A}$ & NA/NA & NSAID & Monocyclic & 0 \\
\hline $\begin{array}{l}\text { 1993, Le Loët } \\
\text { [5] }\end{array}$ & 27 & $20 \mathrm{wg}$ & $\begin{array}{l}\text { A, F, L, } \\
\text { E, Pha, }\end{array}$ & NA/NA & $\begin{array}{l}\text { Prednisone } \\
1 \mathrm{mg} / \mathrm{kg} / \mathrm{d}\end{array}$ & Polycyclic & OHA \\
\hline 1993, Le Loët & 24 & $20 \mathrm{wg}$ & $\begin{array}{c}\text { A, F, L, } \\
\text { E, Pha, } \\
\text { AM }\end{array}$ & NA/NA & $\begin{array}{l}\text { Prednisone } \\
20 \mathrm{mg} / \mathrm{d}\end{array}$ & Polycyclic & 0 \\
\hline $\begin{array}{l}\text { 1994, } \\
\text { Falkenbach } \\
\text { [6] }\end{array}$ & 25 & $8 \mathrm{wg}$ & $\begin{array}{c}\text { A, F, L, } \\
\text { AM, } \\
\text { HSMG, } \\
\text { S, Pha, }\end{array}$ & NA/NA & $\begin{array}{l}\text { High dose } \\
\text { prednisone, } \\
\text { EDX }\end{array}$ & $\begin{array}{c}\text { Polycyclic } \\
\text { (suites de } \\
\text { couches) }\end{array}$ & FE \\
\hline
\end{tabular}




\begin{tabular}{|c|c|c|c|c|c|c|c|}
\hline $\begin{array}{l}\text { 1999, Liozon } \\
\text { [7] }\end{array}$ & 28 & $10 \mathrm{wg}$ & $\begin{array}{l}\text { A, F, L, } \\
\text { E, Pha. }\end{array}$ & ${ }^{A} \mathrm{NA} / \mathrm{NA}$ & RP NA & $\begin{array}{l}\text { Polycyclic } \\
(22 \mathrm{wg}, \\
31 \mathrm{wg}, \text { PP } \\
5 \mathrm{~m})\end{array}$ & $\begin{array}{l}\text { Pre- } \\
\text { eclampsia }\end{array}$ \\
\hline $\begin{array}{l}\text { 2003, Vivien } \\
\text { [8] }\end{array}$ & 21 & $20 \mathrm{wg}$ & $\begin{array}{c}\text { F, AM, } \\
\text { L, S, Pha, } \\
\text { E }\end{array}$ & NA/NA & $\begin{array}{c}\text { Prednisone } \\
1 \mathrm{mg} / \mathrm{kg}+ \\
\text { HCQ }\end{array}$ & $\begin{array}{l}\text { Polycyclic } \\
\text { (23wg, PP) }\end{array}$ & $\begin{array}{c}\text { Prematurity } \\
\text { at } 34 \mathrm{wg} \text {, } \\
\text { IUGR }\end{array}$ \\
\hline 2003, Vivien & 38 & $22 \mathrm{wg}$ & $\mathrm{A}, \mathrm{F}, \mathrm{E}$ & NA/NA & $\begin{array}{l}\text { Prednisone } \\
1 \mathrm{mg} / \mathrm{kg} / \mathrm{d}\end{array}$ & Monocyclic & $\begin{array}{l}\text { None, } 41 \\
\text { wg birth }\end{array}$ \\
\hline 2003, Vivien & 21 & $20 \mathrm{wg}$ & $\begin{array}{l}\text { F, A, } \\
\text { Pha. }\end{array}$ & $\begin{array}{c}33 \\
900 / \mathrm{NA}\end{array}$ & $\begin{array}{l}\text { Prednisone } \\
0.5 \mathrm{mg} / \mathrm{kg} / \mathrm{d}\end{array}$ & Monocyclic & $\begin{array}{l}\text { IUGR, } \\
\text { Prematurity } \\
\text { at } 34 \mathrm{wg}\end{array}$ \\
\hline $\begin{array}{l}\text { 2011, Fischer- } \\
\text { Betz [9] }\end{array}$ & 29 & $12 \mathrm{wg}$ & $\begin{array}{l}\text { F, A, } \\
\text { HSMG, } \\
\text { E, Pha }\end{array}$ & $\begin{array}{c}>40000 / \\
\text { NA }\end{array}$ & $\begin{array}{c}\text { Prednisone } \\
100 \\
\text { mg/day, } \\
\text { Anakinra }\end{array}$ & Polycyclic & $\begin{array}{l}\text { Prematurity } \\
\text { at } 36 \mathrm{wg}\end{array}$ \\
\hline $\begin{array}{l}\text { 2011, } \\
\text { Yamamoto M } \\
\text { [10] }\end{array}$ & 28 & $21 \mathrm{wg}$ & $\begin{array}{l}\text { F, E, A, } \\
\text { AM, L, } \\
\text { S, HLH }\end{array}$ & 24883/NA & $\begin{array}{c}\text { Methylpred } \\
\text { nisone, } \\
\text { Ciclosporin } \\
\text { e }\end{array}$ & Monocyclic & $\begin{array}{l}\text { Prematurity } \\
\text { at } 33 \mathrm{wg} \text {, } \\
\text { IUGR }\end{array}$ \\
\hline $\begin{array}{l}\text { 2013,Hamma } \\
\text { mi S [11] }\end{array}$ & 32 & $22 \mathrm{wg}$ & $\begin{array}{c}\text { F, A, E, } \\
\text { AM, } \\
\text { SMG, S }\end{array}$ & $12957 / \mathrm{NA}$ & $\begin{array}{l}\text { Prednisone } \\
1 \mathrm{mg} / \mathrm{kg}\end{array}$ & Monocyclic & $\begin{array}{l}\text { Prematury } \\
\text { at } 34 \mathrm{wg}\end{array}$ \\
\hline $\begin{array}{l}2014, \\
\text { Mahmoud M } \\
{[12]}\end{array}$ & 25 & $26 \mathrm{wg}$ & $\begin{array}{c}\mathrm{F}, \mathrm{A}, \\
\text { pancytop } \\
\text { enia }\end{array}$ & $1733 / \mathrm{NA}$ & $\begin{array}{l}\text { Prednisone } \\
60 \mathrm{mg} / \mathrm{j}\end{array}$ & Monocyclic & NA \\
\hline $\begin{array}{l}2014, \\
\text { Gerfaud- } \\
\text { Valentin [13] }\end{array}$ & 33 & $10 \mathrm{wg}$ & $\mathrm{F}, \mathrm{A}, \mathrm{E}$ & $3592 / 1 \%$ & $\begin{array}{l}\text { Prednisone } \\
0.7 \mathrm{mg} / \mathrm{kg} / \mathrm{d}\end{array}$ & $\begin{array}{l}\text { Flare at } 1 \\
\text { month PP, } \\
\text { polycyclic } \\
\text { course }\end{array}$ & $\begin{array}{l}\text { Prematurity } \\
\text { at } 34 \mathrm{wg} \text {, } \\
\text { PPRM }\end{array}$ \\
\hline
\end{tabular}




\begin{tabular}{|c|c|c|c|c|c|c|c|}
\hline $\begin{array}{l}2014, \\
\text { Gerfaud- } \\
\text { Valentin }\end{array}$ & 27 & $14 \mathrm{wg}$ & $\begin{array}{l}F, A, \\
\text { SMG, E }\end{array}$ & $4124 / 12 \%$ & $\begin{array}{l}\text { Prednisone } \\
1 \mathrm{mg} / \mathrm{kg} / \mathrm{d}+ \\
\text { Ig IV }\end{array}$ & $\begin{array}{c}\text { Flare at } 1 \text { and } \\
3 \text { month, } \\
\text { polycyclic }\end{array}$ & 0 \\
\hline $\begin{array}{l}2014, \\
\text { Gerfaud- } \\
\text { Valentin }\end{array}$ & 36 & $14 \mathrm{wg}$ & $\begin{array}{l}\text { F, AM, } \\
\text { E, L }\end{array}$ & $1311 / 12 \%$ & $\begin{array}{l}\text { Prednisone } \\
0.5 \mathrm{mg} / \mathrm{kg} / \mathrm{d}\end{array}$ & Monocyclic & $\begin{array}{c}\text { Prematurity } \\
\text { at } 32.5 \mathrm{wg}, \\
\text { PPRM, } \\
\text { OHA }\end{array}$ \\
\hline $\begin{array}{l}2015, \\
\text { Tsuyoshi [33] }\end{array}$ & 32 & $14 \mathrm{wg}$ & $\begin{array}{l}\text { F, L, A, } \\
\text { E, Pha }\end{array}$ & 2920/NA & $\begin{array}{c}\text { Prednisone } \\
1 \mathrm{mg} / \mathrm{kg} / \mathrm{d}, \\
\text { LCAP }\end{array}$ & Monocyclic & $\begin{array}{l}\text { Prematurity } \\
\text { at } 34 \mathrm{wg} \text {, } \\
\text { PPRM }\end{array}$ \\
\hline $\begin{array}{l}\text { 2016, present } \\
\text { case }\end{array}$ & 38 & $12 \mathrm{wg}$ & $\begin{array}{c}\text { F, } \\
\text { HSMG, } \\
\text { A, Pha }\end{array}$ & $\begin{array}{c}41000 /<5 \\
\%\end{array}$ & $\begin{array}{c}\text { Prednisone } \\
1 \mathrm{mg} / \mathrm{Kg} / \mathrm{d} \text {, } \\
\text { Iv IG }\end{array}$ & Polycyclic & $\begin{array}{l}\text { Prematury } \\
\text { at } 34 \mathrm{wg}, \\
\text { fœtal } \\
\text { extraction }\end{array}$ \\
\hline
\end{tabular}

$\mathrm{Wg}$ : weeks of gestation ; A : arthritis, $\mathrm{E}$ : erythema, F : fever, L : lymphadenopathy, S : seritis, AM : arthromyalgia, FE : foetal extraction, Pha : pharyngitis, OHA : oligohydroamnios, SMG : splemomegalia, HSMG : hepatosplenomegalia, IUGR : intrauterine growth restriction, LCAP : leucocytapheresis, PPRM : preterm premature rupture of membranes, Iv IG : intravenous immunoglobulins, PP : post partum. 